\title{
THE CURRENT STATE OF CARTILAGE TRANSPLANTATION \\ IN THE KNEE
}

\author{
Shibu Krishnan, George Bentley \\ Royal National Orthopaedic Hospital \\ shibupkrishnan@hotmail.com
}

\begin{abstract}
Articular hyaline cartilage damage is difficult to repair since the chondrocytes often fail to multiply in vivo. Despite many centuries of advances in medical science the repair of damaged articular cartilage tissue remains controversial. This article presents a comprehensive review of the current state of articular cartilage implantation in the knee.
\end{abstract}

\section{Introduction}

Articular hyaline cartilage damage is difficult to repair since the chondrocytes often fail to multiply in vivo. William Hunter $(1743)^{1}$ famously noted that "From Hippocrates down to the present age, we shall find that an ulcerated cartilage is universally allowed to be a very troublesome disease; that it admits of a cure with more difficulty than a carious bone; and that when destroyed, it is never recovered". Despite two centuries of advances in medical science this statement unfortunately remains true till date.

The incidence of osteo-chondral injury among patients with acute haemarthrosis with a normal clinical and radiographic examination has been estimated to be $20 \%^{2}$. However, small cartilage injuries seen at the time of anterior cruciate ligament reconstruction are recorded as remaining asymptomatic at a mean of 8.7 years $^{3}$.

The natural history of isolated asymptomatic cartilage defects still remain poorly understood. It appears definite that partial thickness cartilage defects will never undergo spontaneous repair whereas full thickness defects can produce fibro-cartilage or fibrous tissue repair which is deficient in type II collagen, has inferior biomechanical characteristics which result in inferior load bearing capacity and predictably short term symptom relief. It still remains unclear as to why some of these full thickness cartilage defects remain asymptomatic for a significant time and it is often thought that the involvement of sub-chondral bone is the source of pain.

The aim of any cartilage repair technique is to produce durable symptomatic and functional benefit ${ }^{1}$, and prevent osteoarthritis in patients with symptomatic chondral/osteochondral defects. A logical solution to achieve durable benefit in patients is to produce hyaline cartilage repair tissue with comparable macroscopic, microscopic and biomechanical characteristics to that of the adjacent native cartilage. Most of the newer treatment modalities aimed at repairing hyaline cartilage defect appear promising in the short term. However, they often fail to result in long-term symptom relief. Autlogous chondrocyte implantation technique appears to be a promising scientific advance in the right direction to cause regeneration of hyaline articular cartilage.

\section{Development of Clinical Autologous Chondrocyte Implantation (ACI)}

Chondrocytes, like most mesenchymal cells fail to multiply in vivo. Attempts currently focus on making them multiply under labora- 
tory conditions and to implant the chondrocytes/chondrocyte-precursor cells into the cartilage defects to affect hyaline cartilage repair. For cartilage implantation to be successful, the chondrocytes must survive preservation and retain their capacity to produce normal matrix components: proteoglycans and Type II collagen.

Audrey Smith (1965) ${ }^{4}$ reported the first successful isolation and freezing of living chondrocytes. It was noted that the frozen-thawed chondrocytes retained viability ex vivo. Bentley and Greer $(1971)^{5}$ reported the first successful chondrocyte allografts (homotransplantation) in rabbits. Kimura et al. $(1984)^{6}$ reported that chondrocytes embedded in collagen gels maintain cartilage phenotype during prolonged culture. It was demonstrated that, under specific culture conditions, chondrocytes assumed a rounded morphology, accumulated metachromatic matrix, and took on the cytological characteristics of the in vivo cartilage cells. Aston and Bentley $(1986)^{7}$ reported on successful cultured chondrocyte allografts without bony components for the repair of chondral defects in rabbits.

Brittberg et al (1994) ${ }^{8}$ reported the first successful regeneration of human osteochondral defects using cultured chondrocytes the autologous chondrocyte implantation technique. Since then, this technique has been evolving with the later development of various matrices and covering membranes.

Autologous chondrocyte implantation procedure: This is done in two stages. The first stage involves a diagnostic arthroscopy when the presence of a chondral defect is confirmed and the suitability of the patient for the procedure is assessed. A small piece of full thickness cartilage is then harvested from a non-

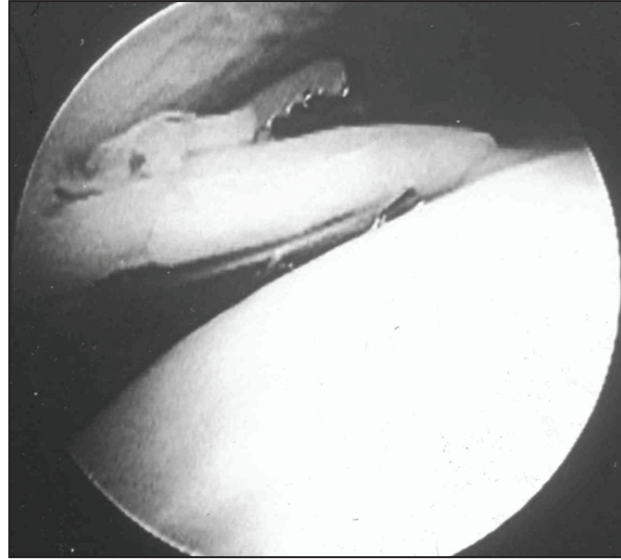

Figure 1. First stage ACI procedure: arthroscopic appearance of harvesting of a full thickness piece of cartilage from the edge of trochlea. (By kind permission of the Journal of Bone and Joint Surgery [Br])

significant part of the knee joint (usually the medial or lateral edges of the trochlea) and the tissue is sent to the laboratory for isolation and culture of chondrocytes.

The second stage involves an open procedure when the cultured chondrocytes are implanted into the defect. The chondrocytes are either injected into the defect after covering the defect with a piece of periosteum (ACI-P)

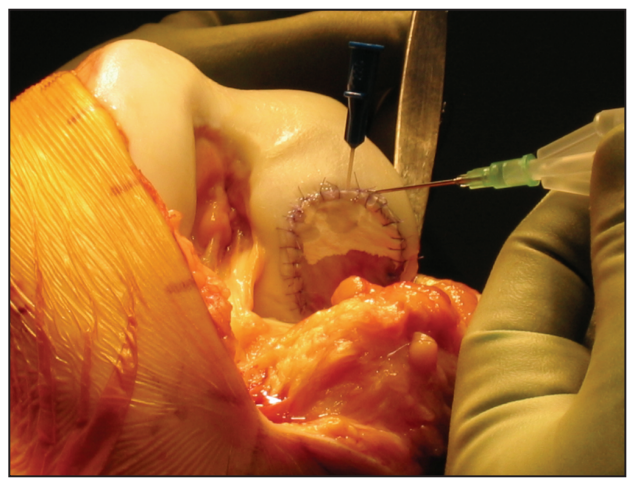

Figure 2. Second stage ACI-C: the collagen membrane is sutured to the edges of the defect and chondrocytes are seen injected beneath the membrane 


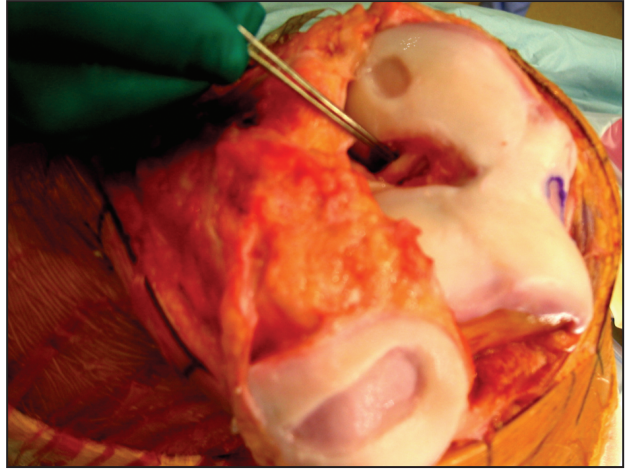

Figure 3. Combined MACI (for three discrete chondral defects) and PCL reconstruction in a patient with multiple post-traumatic chondral defects and PCL deficiency

or type I/III collagen membrane (ACI-C) (Figure 2) which is sutured in position. Alternatively, the cells are stuck down on to the defect using a tissue glue after impregnating them in a bio-synthetic membrane (MACI) (Figure 3).

Our rehabilitation regime includes full weight-bearing mobilisation in a cylinder cast which is removed at the 10 th post-operative day. Patients are then allowed to bend their knees and outpatient physiotherapy is instituted for the first two weeks in order to ensure a full range of motion. All patients are advised to avoid impact loading and twisting movements of the knee.

\section{Stanmore experience of autologous chondrocyte implantation}

We began performing ACI in Stanmore in 1998 and since then have performed this procedure in more than one thousand cases. All these patients are reviewed prospectively at 6 weeks, 6 months, and one year and then on a yearly basis. A check arthroscopy and needle biopsy of the repair tissue is performed at one year, when ever possible.

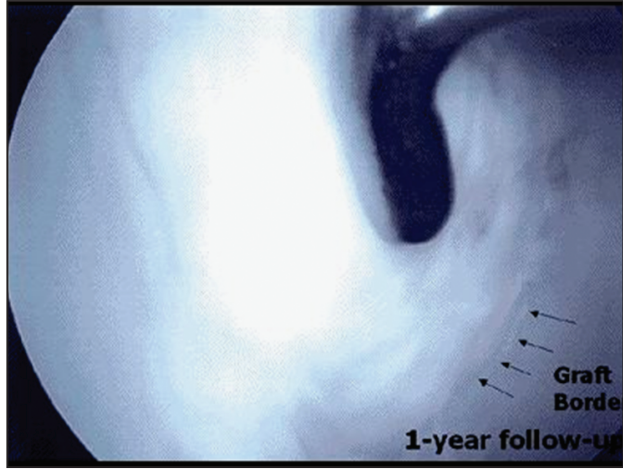

Figure 4. Appearance of repair tissue at I year following ACI - the defect is completely filled with cartilage

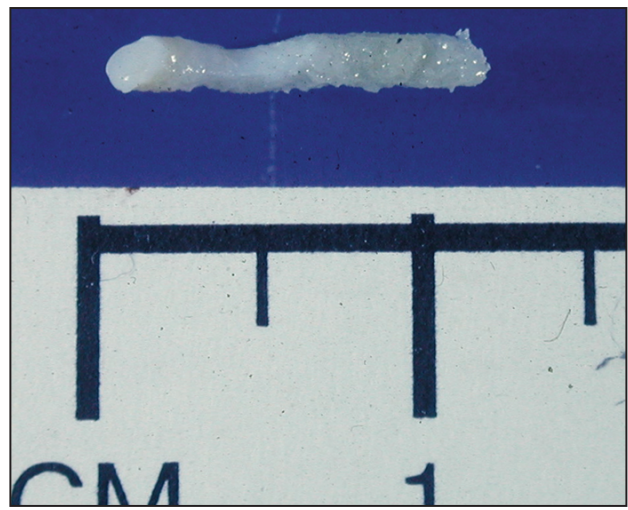

Figure 5. Tissue obtained by needle biopsy from the middle of the repair site

We used initially periosteum as covering membrane (ACI-P) and later used a porcinederived type I/III collagen covering membrane (ACI-C) since it was found to reduce the morbidity of periosteum harvest site without compromising the clinical outcome ${ }^{9}$.

Currently a nationwide multi-centre RCCT comparing ACI-C technique with the MACI (Matrix carried ACI) technique of ACI is underway. In the MACI technique, the cultured chondrocytes are seeded in a bilayered type I/III collagen membrane which has two surfaces: (1) a smooth surface comprising of high density collagen fibres which faces the 
joint and (2) a rough surface where the spaces between the collagen fibres are seeded with chondrocytes. The matrix of MACI remodels after few months and is replaced by the extracellular matrix regenerate. The advantages of MACI technique include a quicker procedure that can be done through a smaller incision and the membrane can cover larger and inaccessible defects with indiscrete margins. There is less damage to surrounding normal cartilage since there is no need for suturing to secure the membrane. There is also no risk of leakage of cells or uneven distribution of cells, unlike in ACI-C. The disadvantages of MACI include a lower number of chondrocytes and the potential for displacement of the graft.

Patients who benefited most with ACI are younger patients (15 to 40 years old) with higher pre-operative modified Cincinnati scores, a less than two-year history of symptoms, those with a single defect, a defect on the trochlea or lateral femoral condyle and patients with fewer than two previous procedures on the index knee ${ }^{10}$. Revision ACI-C in patients with previous ACI and mosaicplasties, which had failed, produced significantly inferior clinical results.

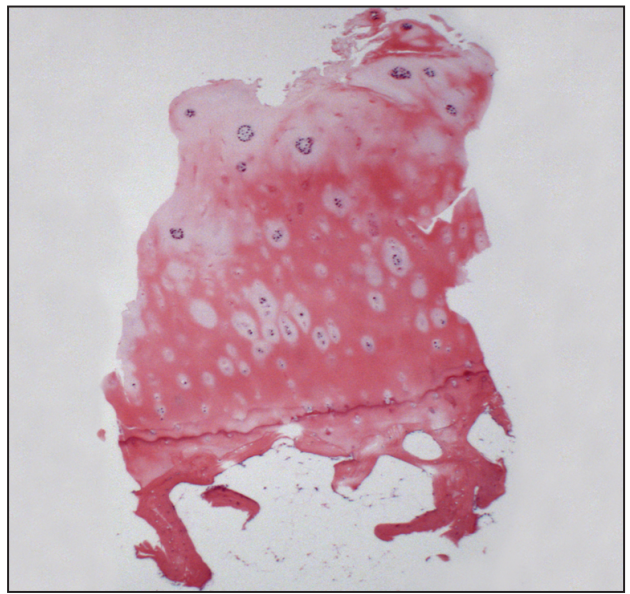

Figure 6. Hyaline-like cartilage repair tissue following ACI: Needle biopsy at 1 year

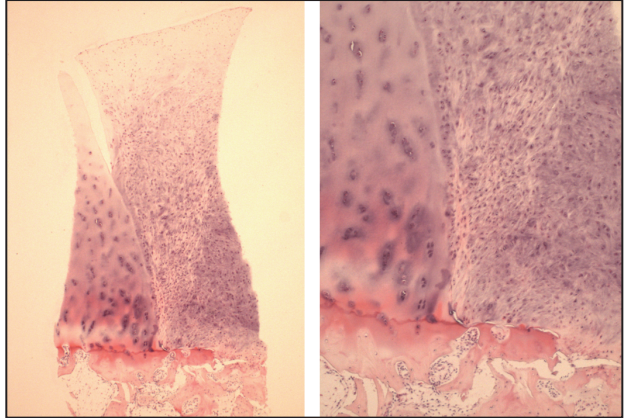

Figure 7. Fibro-cartilage repair tissue with normal adjacent hyaline cartilage: Biopsy at 1 year showing good integration along the rim of repair

RCCT $^{*}$ comparing ACI and mosaicplasty (2003) ${ }^{11}$ : A total of 100 patients with a mean age of 31.3 years (16 to 49 ) and with a symptomatic lesion of the articular cartilage in the knee that was suitable for cartilage repair were randomised to undergo either ACI or mosaicplasty; 58 patients had ACI and 42 mosaicplasty. The mean follow-up was 19 months (12 to 26). Functional assessment using the modified Cincinnati and Stanmore scores and objective clinical assessment showed that $88 \%$ had excellent or good results after ACI compared with 69\% after mosaicplasty. Arthroscopy at one year demonstrated excellent or good repairs in $82 \%$ after ACI and in $34 \%$ after mosaicplasty. This study concluded that the continued use of mosaicplasty in treating chondral defects was of dubious value.

\section{Experience of ACI in patients with osteo-} chondritis dissecans $^{12}$ : Of the 37 patients who had ACI for osteochondritis dissecans, excellent and good clinical outcomes were seen in $82.1 \%$ with juvenile-onset disease whereas only $44.4 \%$ of those with adult-onset disease had a similar result at a mean followup of 4.08 years. The age at the time of ACI$\mathrm{C}$ determined the clinical outcome for juve-

\footnotetext{
* Randomized Controlled Clinical Trial (note of the chief editor)
} 
nile-onset disease $(\mathrm{p}=0.05)$, whereas the size of the defect was the major determinant of outcome in adult-onset disease $(p=0.01)$.

Early results of ACI-C versus MACI technique - prospective RCCT $^{13}$ : 44 patients underwent ACI-C and 47 patients underwent MACI in this prospective RCT. Both treatments resulted in improvement of the clinical score after one year. The mean modified Cincinnati knee score increased by 17.6 in the ACI-C group and 19.6 in the MACI group $(p=0.32)$. Arthroscopic assessments performed after one year showed a good to excellent International Cartilage Repair Society score in $79.2 \%$ of ACI-C and $66.6 \%$ of MACI grafts. Hyaline-like cartilage or hyaline-like cartilage with fibrocartilage was found in the biopsies of $43.9 \%$ of the ACI-C and $36.4 \%$ of the MACI grafts after one year. We concluded that the clinical, arthroscopic and histological outcomes are comparable at one year for both ACI-C and MACI.

The three year results of this trial showed that the proportion of patients with excellent and good mean modified Cincinnati knee score were $63.5 \%$ among the ACI-C group $(n=44)$ and $60.2 \%$ among the MACI group $(n=58)$. These results appear less successful than others reported in the literature. This is due to the strict criteria used for this study and also due to patients of all categories has been accepted and many of these patients have had unsuccessful previous surgery. Long-term results of this trial are currently being evaluated.

\section{Worldwide experience of autologous chondrocyte implantation}

Peterson et al $(2000)^{14}$ reported the clinical, arthroscopic, and histological results from the first 101 patients who underwent ACI in Sweden since 1987 for the treatment of large $\left(1.5-12.0 \mathrm{~cm}^{2}\right)$ full thickness chondral defects of the knee at 2 to 9 year follow-up. Good or excellent clinical results were seen in individual groups as follows: Isolated femoral condyle (92\%), multiple lesions (67\%), osteochondritis dissecans (89\%), patella (65\%), and femoral condyle with anterior cruciate ligament repair (75\%). Arthroscopic findings in 53 evaluated patients showed good repair tissue fill, good adherence to underlying bone, seamless integration with adjacent cartilage, and hardness close to that of the adjacent tissue. However, hypertrophic response of the periosteum or graft or both was identified in 26 arthroscopies (49\%); seven were symptomatic and resolved after arthroscopic trimming. Graft failure occurred in seven (four of the first 23 and three of the next 78) patients. Histological analysis of 37 biopsy specimens showed a correlation between hyaline-like tissue (hyaline matrix staining positive for Type II collagen and lacking a fibrous component) and good or excellent clinical results.

Knutzen et al (2007) ${ }^{15}$ in a prospective RCCT of 80 patients, compared micro-fracture with the autologous chondrocyte implantation technique and reported significant improvement in clinical results among both groups at two and five years and found no significant advantage of one technique over the other at five years from the primary procedure. Further long-term follow-up was recommended to determine if one method is better than the other. They also found no significant difference in the histological features of regenerate tissue in either group.

Saris et al (2008) ${ }^{16}$ reported the results of a multi-centre RCT at one year where they compared the clinical and histological outcome of 57 patients who underwent "characterised" chondrocyte implantation against 61 patients who underwent micro-fracture. In "characterised" chondrocyte implantation, 
they used an autologous cartilage cell therapy product that has been optimised for its biological potency to form stable cartilage tissue in vivo. At one-year evaluation they found that the chondrocyte phenotype and tissue structure were superior in the "characterised" chondrocyte implantation group compared with the micro-fracture group. However, there was no significant difference in the clinical outcome between the groups. They concluded that the superior structural outcome might result in improved long-term clinical benefit with "characterised" chondrocyte implantation.

\section{Cost-effectiveness of ACI}

Clar C et al (2005) ${ }^{17}$ conducted a systematic review and economic evaluation of the costeffectiveness of ACI and concluded that ACI could be cost-effective when compared to microfracture technique only if it could be shown that ACI is more likely to produce hyaline cartilage which would in turn be more likely to be durable and to prevent osteoarthritis in the longer term (e.g. 20 years). There is at present insufficient data to conclude that ACI is cost-effective compared with microfracture or mosaicplasty.

\section{Reported complications}

Niemeyer P et al $(2008)^{18}$ in a case series of 309 patients with 349 ACI procedures of the knee identified four major complications which comprised $88.5 \%$ of reasons for revision surgery among 52 patients at a mean follow up of 4.5 years (standard deviation, \pm 1.5 ). They were 1. graft hypertrophy 2. integration insufficiency 3 . insufficient regenerate and 4 . graft delamination. The overall complication rate was highest in the group of patients treated with periosteum-covered ACI as opposed to those who had Chondrogide or membranecovered ACI as covering membrane. The incidence of complications in this series was $14.9 \%$.
Wood et al (2006) ${ }^{19}$ reviewed the adverse events with the "Carticel" implantation that were reported to the Food and Drug Administration agency (FDA) in the United States between 1996 and 2003. A total of 497 adverse events among 294 patients receiving Carticel were reported. The median interval from Carticel implantation to the diagnosis of an adverse event was 240 days (range, one to 2105 days). The most commonly reported adverse events were graft failure (25\%), delamination (22\%), and tissue hypertrophy (18\%). However, based on the manufacturer's reported distribution of 7500 Carticel lots between 1995 and 2002, only 285 patients (3.8\%) had an adverse event that was reported to the Food and Drug Administration.

\section{Recent advances in cartilage repair strategy}

Current cartilage repair strategies fail to produce reproducible levels of long-lasting hyaline cartilage tissue that meets the functional demands placed upon this tissue in vivo in all cases. The reasons for this can be attributed to matrix degradation, differentiation or integration insufficiencies, or loss of the transplanted cells and tissues.

Current techniques include the transplantation of a fully "in vitro" differentiated construct or a graft containing a homogeneous population of rather undifferentiated cells and signalling molecules, to ensure that the desired differentiation process takes place in vivo under physiological conditions of mechanical loading. Each approach has its advantages and disadvantages ${ }^{20}$. The former allows chondrogenic differentiation under controlled in vitro conditions, but is often associated with biocompatibility and integration problems. The latter promotes integration well, but presents the risk of uncontrolled and undesired differentiation processes to occur. 
The ultimate strategy should aim at engineering cartilage constructs by combining cells, scaffold materials and environmental factors, including growth factors, signalling molecules, and physical influences.

\section{Role of bone morphogenetic proteins (BMP) and stem cells in cartilage repair}

Bone morphogenetic proteins are multifunctional growth factors belonging to Transforming Growth Factor Beta (TGF- $\beta$ ) super family. BMP signalling is required both for the formation of pre-cartilaginous condensations from the mesenchymal precursors and for the differentiation of precursors into chondrocytes.

BMPs 2, 4, 6, 7, 9 and 13 stimulate the synthesis of cartilage matrix constituents, type II collagen and aggrecan by adult chondrocytes and therefore have the potential to accelerate and modify cartilage repair in ACI.

The use of BMPs in cartilage repair has not been tested in humans to date and the available studies are all animal experiments. Enhanced BMP activity has been reported in tumour cells and concerns on their potential for tumour genesis require clarification.

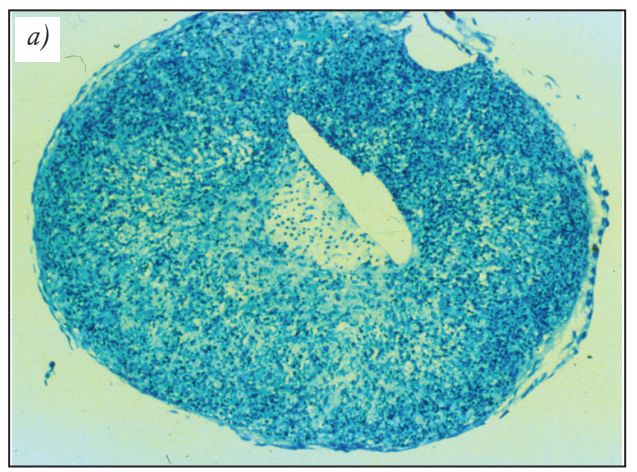

The local delivery of BMPs by geneticallyengineered stem cells has been shown to enhance chondrogenesis and repair of articular cartilage in animals ${ }^{1}$. Stem cells form alternative source of chondrocytes and could avoid the first stage of the current two-stage procedure of ACI and prevent donor site morbidity. BMP4 and other BMPs can induce embryonic stem cells and mesenchymal progenitor cells to undergo chondrogenesis. The mesenchymal derived stem cells (MDCs) induce cartilage repair similar to ACI within 4 wks after transplantation in vivo. This do not require the use of collagen scaffolds (unlike ACI) and are currently being investigated. BMP4-transduced MDSCs can promote either cartilage or bone formation as an end point, depending upon the culture conditions. The site of implantation in vivo also determines whether cartilage or bone repair occurs.

Wilke et al (2007) ${ }^{21}$ injected an autogenous fibrin vehicle containing MSCs or fibrin alone as control into twelve full thickness cartilage defects in 6 young mature horses. Arthroscopic second look and biopsies at 30 days and 8 months were performed. They concluded that the MSCs improved early healing response, but did not significantly enhance the long-term histologic appearance

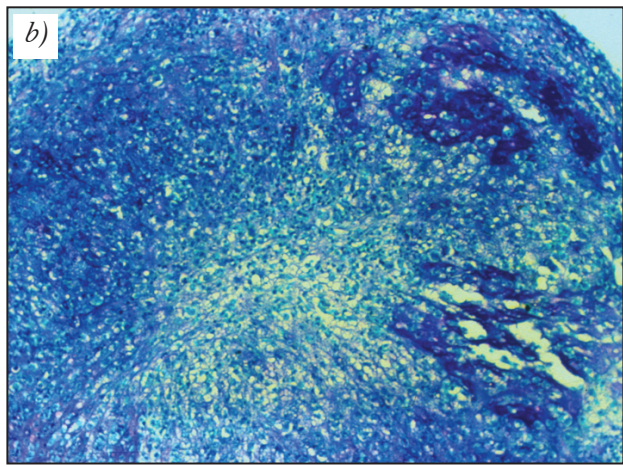

Figure 8. Pellet culture showing human chondrocytes at 14 days (a) control and (b) shows increased cells and matrix in culture treated with BMP (Published with the kind permission of Mr. A Goldberg) 
or biochemical composition of full thickness cartilage lesions. Much further work is needed to test this method.

\section{Gene therapy in cartilage repair}

Gene therapy involves the transfer of genes encoded with specific growth factors into chondrocytes or progenitor cells. For gene expression, the transferred DNA material must enter the nucleus where it can be transcripted and this result in the generation of m-RNA which is then transported outside the nucleus, and serves as a matrix for the production of growth factors in the ribosome. Successful gene transfer of IGF-1 and TGFBeta into chondrocytes, in monolayer cultures and on the surfaces of cartilage explants, has been shown to increase matrix biosynthesis and to maintain the chondrocyte phenotype, especially the production of collagen type-II.

Mason et al $(2000)^{22}$ combined the techniques of gene therapy and tissue engineering to produce cartilage repair tissue. Rabbit mesenchymal stem cells were transduced with retroviral vectors encoded for the gene of BMP-7. These were then seeded on polymer scaffold grafts and implanted into osteochondral defects in rabbit knees.

The major concerns in gene therapy are the safety of the gene transfer and the control of gene expression. Vectors integrating into the genome of the cells bear the risk of insertional mutagenesis and the development of a malignancy or an abnormal regulation of cell growth and toxicity because of chronic over-expression of the growth factor. Pharmacologically-controlled gene expression appears to be a promising strategy to control the timing and level of gene expression.

\section{Potential areas of improvement in cartilage repair strategy}

The current problems with ACI technique include a prolonged rehabilitation period because of the two stage surgical procedure and the time taken for graft maturation; and an unpredictable line of differentiation of the implanted cells into hyaline cartilage, fibrocartilage or fibrous tissue. This is possibly influenced by the implantation site environment, loading characteristics, availability of growth factors, the influence of the biodegradable matrices on cell differentiation, migration, matrix production etc., and the distribution and concentration of chondrocytes or precursor cells in the scaffold.

Emerging techniques to address these problems include the use of arthroscopic implantation of cells thus avoiding the need for arthrotomy for the second stage procedure, the use of stem cells or minced cartilage in various scaffolds in an attempt to make this a single stage procedure, harvesting of cells from the defect margin to prevent donor site morbidity, the use of combination techniques such as ACI with microfracture and the use of "characterised" cells (by gene marking) to achieve quality control in the type of cells used for repair.

\section{Summary}

The ideal candidate for ACI appears to be younger patients (15 to 40 years old) with higher pre-operative modified Cincinnati scores, a less than two-year history of symptoms, those with a single defect, a defect on the trochlea or lateral femoral condyle and patients with fewer than two previous procedures on the index knee. Patients with a high body mass index, smokers and those who had revision ACI-C following previous failed ACI and mosaicplasties produced significantly inferior clinical results. 


\section{Conclusion}

Adults with symptomatic osteochondral defects in the knee often deteriorate and require surgical intervention for severe pain and may develop disabling osteoarthritis. Currently there is no evidence to justify treatment of asymptomatic osteochondral defects smaller that $1 \mathrm{~cm}$ in diameter. Micro-fracture appears a cost-effective procedure for smaller well-contained lesions whereas ACI is successful in $70 \%$ to $80 \%$ of larger lesions in the short to medium term. Much work needs to be done to control and or enhance maturation of implanted condrocytes or chondrocyte-precursor cells to produce repair tissue with durable clinical improvement. Joint instability and mal-alignment should be corrected in order to produce optimum outcome while attempting repair of damaged articular cartilage. New technologies such as the use of stem cells, genetic engineering and gene therapy appear promising but require extensive laboratory and translational research.

\section{REFERENCES}

1. Bentley G. The Cellular Approach to Early joint damage. Robert Jones lecture of the Royal College of Surgeons of England at British Orthopaedic Association Annual meeting: 2007 Sep 28.

2. Noyes FR, Bassett RW, Grood ES, Butler DL. Arthroscopy in acute traumatic haemarthrosis of the knee. Incidence of anterior cruciate tears and other injuries. J Bone Joint Surg Am. 1980 Jul;62(5):687-95, 757.

3. Shelbourne KD, Jari S, Gray T. Outcome of untreated traumatic articular cartilage defects of the knee: a natural history study. J Bone Joint Surg Am. 2003;85-A Suppl 2:8-16.

4. Smith $A U$. Survival of Frozen Chondrocytes Isolated from Cartilage of Adult Mammals. Nature 1965;205:782-4.

5. Bentley G, Greer RB. Homotransplantation of isolated epiphyseal and articular chondrocytes into joint surfaces. Nature, 1971;(230): 385-8.

6. Kimura T, Yasui N, Ohsawa S, Ono K. Chondrocytes embedded in collagen gels maintain cartilage phenotype during long-term cultures. Clin Orthop Relat Res. 1984 Jun;(186):231-9.

7. Aston JE, Bentley G. Repair of articular surfaces by allografts of articular and growth-plate cartilage. J Bone Joint Surg Br. 1986 Jan;68(1): 29-35.
8. Brittberg M, Lindahl A, Nilsson A, Ohlsson C, Isaksson $O$, Peterson $L$. Treatment of deep cartilage defects in the knee with autologous chondrocyte transplantation. N Engl J Med. 1994 Oct 6;331(14):889-95.

9. Gooding CR, Bartlett W, Bentley G, Skinner JA, Carrington $R$, Flanagan A. A prospective, randomised study comparing two techniques of autologous chondrocyte implantation for osteochondral defects in the knee: Periosteum covered versus type I/III collagen covered. Knee 2006 Jun;13(3):203-10.

10. Krishnan SP, Skinner JA, Bartlett W, Carrington $R W$, Flanagan AM, Briggs $T W$, Bentley $G$. Who is the ideal candidate for autologous chondrocyte implantation? J Bone Joint Surg Br. 2006 Jan;88(1):61-4.

11. Bentley G, Biant LC, Carrington RW, Akmal M, Goldberg A, Williams AM, Skinner JA, Pringle J. A prospective, randomised comparison of autologous chondrocyte implantation versus mosaicplasty for osteochondral defects in the knee. J Bone Joint Surg Br. 2003 Mar; 85(2): 223-30.

12. Krishnan SP, Skinner JA, Carrington $R W$, Flanagan AM, Briggs TW, Bentley $G$. Collagencovered autologous chondrocyte implantation for osteochondritis dissecans of the knee: twoto seven-year results. J Bone Joint Surg Br. 2006 Feb;85(2):223-30. 
13. Krishnan SP, Skinner JA, Carrington $R W$, Flanagan AM, Briggs TW, Bentley G. Collagencovered autologous chondrocyte implantation versus matrix-induced autologous chondrocyte implantation for osteochondral defects of the knee: a prospective, randomised study. J Bone Joint Surg Br. 2005 May;87(5):640-5.

14. Peterson L, Minas T, Brittberg M, Nilsson A, Sjögren-Jansson E, Lindahl A. Two- to 9-year outcome after autologous chondrocyte transplantation of the knee. Clin Orthop Relat Res. 2000 May;(374):212-34.

15. Knutsen G, Drogset JO, Engebretsen L, Grøntvedt T, Isaksen V, Ludvigsen TC, Roberts S, Solheim E, Strand T, Johansen $O$. A randomized trial comparing autologous chondrocyte implantation with microfracture. Findings at five years. J Bone Joint Surg Am. 2007 Oct;89(10): 2105-12.

16. Saris DB, Vanlauwe J, Victor J, Haspl $M$, Bohnsack M, Fortems Y, Vandekerckhove B, Almquist KF, Claes T, Handelberg $F$, Lagae $K$, van der Bauwhede J, Vandenneucker H, Yang $K G$, Jelic $M$, Verdonk R, Veulemans $N$, Bellemans J, Luyten FP. Characterized chondrocyte implantation results in better structural repair when treating symptomatic cartilage defects of the knee in a randomized controlled trial versus microfracture. Am J Sports Med. 2008 Feb; 36(2):235-46.

17. Clar C, Cummins E, McIntyre L, Thomas S, Lamb J, Bain L, Jobanputra P, Waugh N. Clinical and cost-effectiveness of autologous chon- drocyte implantation for cartilage defects in knee joints: systematic review and economic evaluation. Health Technol Assess. 2005 Dec; 9(47):iii-iv, ix-x, 1-82.

18. Niemeyer P, Pestka JM, Kreuz PC, Erggelet C, Schmal H, Suedkamp NP, Steinwachs M. Characteristic complications after autologous chondrocyte implantation for cartilage defects of the knee joint. Am J Sports Med. 2008 Nov; 36(11): 2091-9.

19. Wood JJ, Malek MA, Frassica FJ, Polder JA, Mohan AK, Bloom ET, Braun MM, Coté TR. Autologous cultured chondrocytes: adverse events reported to the United States Food and Drug Administration. J Bone Joint Surg Am. 2006 Mar; 88(3):503-7.

20. Steinert AF, Ghivizzani SC, Rethwilm A, Tuan $R S$, Evans $C H$, Nöth $U$. Major biological obstacles for persistent cell-based regeneration of articular cartilage. Arthritis Res Ther. 2007; 9(3):213.

21. Wilke M, Nydam D, Nixon A. Enhanced early chondrogenesis in articular defects following mesenchymal stem cell implantation in an equine model. J Orthop Res. 2007 Jul;25(7): 913-25.

22. Mason JM, Breitbart AS, Barcia M, Porti D, Pergolizzi RG, Grande DA. Cartilage and bone regeneration using gene-enhanced tissue engineering. Clin Orthop Relat Res. 2000 Oct;(379 Suppl):S171-8.

\section{Shibu Krishnan}

Royal National Orthopaedic Hospital

HA7 4LP, Brockley Hill, Stanmore, Middlesex, UK

Tel.: (+44) 020 8954-2300 